\title{
PERCEPÇÃO MACROECONÔMICA, FELICIDADE E IDEOLOGIA: UM ESTUDO DE CASO DO BRASIL (2010-2016) ${ }^{1}$
}

Anderson Henrique ${ }^{2}$

\section{Resumo}

Qual é o efeito da percepção macroeconômica de inflação e desemprego sobre o nível de felicidade? O argumento principal deste trabalho é de que o efeito da percepção da inflação e do desemprego sobre o nível felicidade depende da ideologia política no Brasil. Portanto, testarei duas hipóteses de pesquisa: (1) quanto mais à esquerda estão os indivíduos, quando há percepção de desemprego, menor será o grau de felicidade dos eleitores e (2) quanto mais à direita estão os indivíduos, juntamente com a percepção de inflação, menor será o nível de felicidade. Metodologicamente, o desenho de pesquisa utiliza de estatística descritiva e Regressão Logística para analisar dados secundários do Latin American Public Opinion Project (LAPOP), no período de 2010 a 2016. Os resultados concluem que não há efeito estatisticamente significativo da percepção macroeconômica de inflação e desemprego, condicionado à ideologia, sobre o nível de felicidade dos eleitores no Brasil. Todavia, este estudo corrobora com os achados de opinião pública sobre a insuficiência da ideologia como balizador das escolhas e comportamentos políticos.

Palavras-chave: Felicidade, Ideologia, Percepção Econômica.

\section{Abstract}

What is the effect of macroeconomic perception of inflation and unemployment on the level of happiness? The main argument of this paper is that the effect of the perception of inflation and unemployment on the happiness level depends on the political ideology in Brazil. Therefore, I will test two research hypotheses: (1) the farther left individuals are, when unemployment is perceived, the lower the voters' happiness, and (2) the farther right individuals are, along with the perception of unemployment. inflation, the lower the level of happiness. Methodologically, the research design uses descriptive statistics and logistic regression to analyze secondary data from the Latin American Public Opinion Project (LAPOP), from 2010 to 2016. The results conclude that there is no statistically significant effect on macroeconomic perception of inflation and unemployment, conditioned by ideology, on the level of voter happiness in Brazil. However, this study corroborates the findings of public opinion on the insufficiency of ideology as a marker of political choices and behaviors.

Keywords: Happiness, Ideology, Economic Perception.

\footnotetext{
${ }^{1}$ Agradeço as contribuições dos professores Marcus André Melo (UFPE), Dalson Britto (UFPE), Mariana Batista (UFPE), Davi Moreira (FGV-SP), aos pareceristas da revista e a Antônio Fernandes (UFPE), que contribuíram para a confecção final deste artigo.

${ }^{2}$ Graduado e mestrando em Ciência Política pela Universidade Federal de Pernambuco (UFPE). Atualmente é bolsista da Fundação de Amparo à Ciência e Tecnologia de Pernambuco (FACEPE) e integra os grupos de pesquisa "Métodos de Pesquisa em Ciência Política" e "Instituições, Políticas e Governo", ambos da UFPE. E-mail: andersonheri@gmail.com.
} 


\section{Resumen}

¿Cuál es el efecto de la percepción macroeconómica de la inflación y el desempleo en el nivel de felicidad? El argumento principal de este artículo es que el efecto de la percepción de la inflación y el desempleo en el nivel de felicidad depende de la ideología política en Brasil. Por lo tanto, probaré dos hipótesis de investigación: (1) cuanto más lejos están los individuos que quedan, cuando se percibe el desempleo, menor es la felicidad de los votantes, y (2) más lejos están los individuos de la derecha, junto con la percepción del desempleo. inflación, cuanto más bajo es el nivel de felicidad. Metodológicamente, el diseño de la investigación utiliza estadística descriptiva y regresión logística para analizar datos secundarios del Proyecto de Opinión Pública de América Latina (LAPOP), de 2010 a 2016. Los resultados concluyen que no hay un efecto estadísticamente significativo en la percepción macroeconómica de la inflación y el desempleo, condicionado por la ideología, en el nivel de felicidad de los votantes en Brasil. Sin embargo, este estudio corrobora los hallazgos de la opinión pública sobre la insuficiencia de la ideología como marcador de elecciones y comportamientos políticos.

Palabras-clave: Felicidad, Ideología, Percepción económica.

\section{INTRODUÇÃO}

Qual é o efeito da percepção macroeconômica de inflação e desemprego sobre o nível de felicidade? O argumento principal deste trabalho é de que o efeito da percepção da inflação e do desemprego sobre o nível felicidade depende da ideologia política no Brasil. Portanto, estabeleço duas hipóteses: (1) quanto mais à esquerda estão os indivíduos, quando há percepção de desemprego, menor será o nível de felicidade dos eleitores; e (2), quanto mais à direita estão os indivíduos, juntamente com maior percepção de inflação, menor será o nível de felicidade.

A literatura sobre desempenho econômico e felicidade mostra que, empiricamente, indivíduos que se localizam mais à esquerda do espectro ideológico se importam mais com o desemprego em relação à inflação do que os de direita (FREY E SCHNEIDER, 1978). Os trabalhos sobre felicidade partidária buscam mensurar a relação entre variáveis macroeconômicas e ideologia do governo no nível de felicidades dos cidadãos (DI TELLA, MAC CULLOCH E OSWALD, 2001; 2003, DI TELLA, 2005).

Em relação à ideologia, o debate não é consensual sobre o seu efeito na política. Autores clássicos de opinião pública, acreditavam que a ideologia também teria um papel primordial em orientar e servir com um atalho cognitivo de como os indivíduos guiariam suas preferências políticas e escolha do voto (CAMPBELL et al., 1960). Outros autores, encontraram o contrário, que a ideologia seria complexa (STOKES, 1960; DAVIS et al., 1970), confusa (CONSERVE, 1964) e não-informativa (CONSERVE E PIERCE, 1986) para o eleitor tomar sua decisão na escolha do 
voto, principalmente para vários países na América Latina (ZECHMEISTER, 2015) e com resultados que divergem no Brasil (CARREIRÃO, 2002; 2007; IZUMI, 2019).

A originalidade deste artigo repousa na inserção da variável ideológica como um dos determinantes da felicidade no Brasil. Os resultados sugerem que a percepção de problemas macroeconômicos, quando levado em consideração a ideologia dos indivíduos, não impacta na felicidade subjetiva dos eleitores no Brasil.

\section{A RELAÇÃO ENTRE PERFORMANCE ECONÔMICA DO GOVERNO E A FELICIDADE PARTIDÁRIA}

Os estudos sobre o impacto da performance política na economia vêm sendo debatidos pela Ciência Política por muito tempo, buscando analisar como a ideologia dos governos poderiam produzir diferentes políticas econômicas (HIBBS,1977; ALESINA, 1987). Muitos desses trabalhos buscaram analisar como os partidos políticos poderiam produzir políticas que privilegiariam determinados assuntos econômicos, como diminuir os índices de inflação e a taxa de desemprego, e que esses poderiam ser bom preditores para inclinação partidária.

Frey e Schneider (1978) analisaram a importância das variáveis macroeconômicas na política e encontraram que elas exercem influência na votação e nos resultados políticos. Por outro lado, Roubin (1998) encontrou evidências claras dos ciclos eleitorais sobre a adoção de políticas, as quais influenciam as taxas de inflação nos países, fruto de um modelo oportunista. Esses trabalhos sugerem que o agregado macroeconômico de fato importa para as pessoas.

Para Di Tella (2005), os achados de estudos como de Frey e Schneider (1978), Roubin (1992) e outros contemporâneos de sua época, convergiram para dois vieses. Os primeiros achados dizem respeito à dificuldade de se construir testes formais capazes de identificar as preferências políticas dos policymakers, uma vez que os resultados e escolhas políticas se diferenciariam por países e governos. Além da falta de disponibilidade de dados para elaborar estudos comparados robustos.

O segundo resultado desses estudos tem foco na performance das políticas dos modelos partidários. Di Tella (2005) afirma que os testes estatísticos elaborados seriam inconclusivos, tendendo a focar apenas no modelo partidário ao invés dos modelos oportunistas, especialmente, nos resultados econômicos ${ }^{3}$.

\footnotetext{
${ }^{3}$ Frey e Schneider (1978a, b), Golden e Poterba (1980), Hibbs (1987) e Chappell, Havrilesky e McGregor (1993).
} 
Ambas as conclusões desses trabalhos teriam foco mais no modelo partidário do que nos ciclos políticos sobre performance econômica dos governos. Ainda, segundo o autor, começou-se a analisar impacto e a importância que essas ações macroeconômicas teriam no comportamento dos indivíduos, em especial, na felicidade (DI TELLA, 2005).

Pouco se sabia, empiricamente, sobre o efeito das flutuações macroeconômicas sobre o bem-estar humano (DI TELLA, MAC CULLOCH E OSWALD, 2001; 2003). Segundo Di Tella, Mac Culloch e Oswald (2001), os economistas utilizaram com menos frequência, comparativamente, os dados de bem-estar nos questionários. Dos poucos trabalhos, Easterlin (1974) encontrou que a renda estaria associada com os altos níveis de felicidade entre os indivíduos nos Estados Unidos, mas que ao analisar no tempo, não haveria maiores ganhos de bem-estar pelo aumento da renda, chamando esse fenômeno de "Paradoxo de Easterlin". Por sua vez, Di Tella, Mac Culloch e Oswald (1997) encontraram uma relação significativa entre renda, ano, países e o sentimento de felicidade e que esse efeito diferiria entre os países da OCDE.

Posteriormente, Di Tella, MacCulloch e Oswald (2003) analisaram os dados de bem-estar nos surveys dos países Europeus e apontaram que haveria fortes padrões microeconômicos nos dados e que esses seriam semelhantes para vários países. Assim, os dados de felicidade se comportariam de forma previsível. Segundo os autores, características pessoais, análises por países, no tempo e forças macroeconômicas impactariam estatisticamente no bem-estar da população (DI TELLA, MACCULLOCH E OSWALD, 2003).

Desenvolvendo esses achados, Di Tella e MacCulloch (2005) utilizaram modelos mais robustos e mensuram como o efeito dos aspectos partidário das políticas macroeconômicas (inflação e desemprego) afetariam o grau de felicidade. Nesse trabalho, os autores concluíram que haveria diferença significativa entre a percepção de felicidade entre as pessoas de direita e esquerda, quando analisados em cada país por efeitos fixos e anos. Isto é, pessoas de direita se preocupam mais com inflação e de esquerda com o desemprego (DI TELLA E MACCULLOCH, 2005).

Ademais, chegam aos resultados de um trade-off no ciclo político-econômico, em que, quando o governo se inclina ideologicamente para a direita, se preocupando mais com política de combate à inflação, os indivíduos de direita apresentariam maiores pontuações de felicidade. Em contrapartida, no mesmo período, os indivíduos de esquerda se mostram menos felizes. Portanto, para os autores, o governo afetaria o bem-estar dos seus eleitores, sendo expresso pelas políticas macroeconômicas de desemprego e inflação.

Apesar dos achados, Di Tella e MacCulloch (2005) acreditam que há duas explicações para os resultados. O primeiro repousa na ideia de existência de políticas não ligadas à macroeconomia, 
nas quais governo diferem, como política agrícola, combate ao crime organizado e políticas ligadas às questões sociais. O segundo gira na função de utilidade, na qual a variável posição ideológica do governo (governo de direta, por exemplo) é fortemente correlacionado com a inflação (negativamente) e o desemprego (positivamente).

No mesmo caminho, analisando o efeito da felicidade na política, trabalhos como de Singh (2004) estima a satisfação dos eleitores com o partido vencedor em democracias, quando os eleitores fazem escolhas ótimas (o partido vencedor é sua primeira intenção de voto) e sub ótima (a escolha do candidato não corresponde a primeira opção de voto).

O autor conclui o trabalho mostrando que a vitória ótima tem um efeito maior na satisfação, quando a otimização da escolha do voto é conceituada em termos de similaridade e identificação partidária, do que quando conceituada em termos de proximidade ideológica. Além disso, mostra que os arranjos institucionais podem gerar voto sincero e uma população satisfeita (SINGH, 2004).

Em artigo posterior, Pinto et al. (2019) buscam examinar o efeito dos resultados das eleições presidenciais de 2012 e 2016 nos Estados Unidos para o bem-estar subjetivo dos Democratas e Republicanos, avaliando a felicidade em dois aspectos em relação à satisfação da vida e hedônica. Os autores abordam como os recentes resultados das eleições presidenciais dos Estados Unidos foram impactados pela performance da economia e da insatisfação dos eleitores para com os partidos políticos.

Segundo esses autores, os indivíduos com mais satisfação em suas vidas tendem a votarem e participar do processo político com demonstrações públicas nos EUA. Ainda segundo eles, os estudos de participação política e felicidade encontraram dois efeitos: (1) indivíduos que são mais satisfeitos com suas vidas são mais susceptíveis a votarem e; (2) que a ação de votar por si só pode ter um efeito positivo sobre pesquisas, sendo mais sobre as eleições do que a satisfação dos indivíduos (PINTO et al.,2019).

\section{OS DETERMINANTES EMPÍRICOS DO BEM-ESTAR SUBJETIVO NO BRASIL}

No Brasil, pesquisas sobre felicidade se concentram em estudos econométricos, buscando identificar em que medida as variáveis socioeconômicas, como renda, desemprego, educação e estado civil afetam no nível de felicidade dos indivíduos (CAVALCANTI et al.,2009; ISLAM et al. 2009; LUZ et al., 2011).

Corbi e Menezes-Filho (2006) fazem o primeiro esforço empírico de mensurar quais seriam os determinantes da felicidade no Brasil, utilizando dados do World Values Survey (WVS) para mais 
quatro países, buscam analisar como variáveis econômicas de renda e desemprego podem afetar o bem-estar individual ao longo do tempo. Os autores concluem uma relação positiva e significativa entre felicidade e renda para os países analisados e que pessoas mais ricas e com empregos teriam mais chance de serem felizes.

Por outro lado, em um estudo comparado usando modelos logit, entre os estudantes de Economia da Universidade de Federal de Pernambuco (UFPE) e a da Purdue University nos Estados Unidos, Cavalcanti et al. (2009) encontram diferença significativa entre o bem-estar individual para os dois grupos. Segundo esses autores, as mulheres estudantes seriam mais felizes que os homens na Purdue, os homens da UFPE seriam mais felizes, a base familiar não teria impacto sobre a felicidade em Purdue, sendo significante na UFPE e que o dinheiro extra seria significante em ambos os grupos.

Apesar desses resultados que relacionam positivamente renda e bem-estar subjetivo, Cobi e Menezes-Filho (2006), Cavalcanti et al. (2009) e outros autores, carecem de uma explicação mais profunda do bem-estar subjetivo levando em consideração os determinantes sociais e políticos.

Ribeiro (2015) avança no debate ao inserir nos modelos explicativos não só fatores de renda, como também a percepção e relação social associados à felicidade. Para o autor, não apenas condições materiais estariam atreladas ao nível de satisfação, mas também fatores ligados às relações pessoais e as percepções sobre renda e saúde seriam fundamentais para explicar o grau de felicidade no Brasil. De acordo com os resultados, Ribeiro (2015) encontra que a renda, saúde e religiosidade são fatores que aumentam a probidade de um indivíduo ser feliz. Além disso, mostra que companheirismo, relações sociais de amizade, casamento, sensação de segurança e satisfação com a saúde estão positivamente relacionadas com o aumento significativo do bem-estar subjetivo.

Os achados empíricos que esses estudos apresentam são importantes para entender a variação do nível de satisfação individual dos cidadãos brasileiros nos aspectos sociais e econômicos. Entretanto, tais trabalhos sobre o Brasil, ausentam-se de incluir em suas análises variáveis relacionadas à ideologia, sendo essa a principal contribuição desenvolvida neste artigo.

\section{OPINIÃO PÚBLICA E IDEOLOGIA: OS EFEITOS NAS ESCOLHAS POLÍTICAS}

A estrutura ideológica foi considerada um atalho cognitivo primordial utilizado pelo indivíduo para organizar seus pensamentos em torno da política e economia (CONVERSE, 1964). Campbell et al. (1960) na obra clássica "The American Voter", forneceram uma análise profunda sobre o papel da ideologia na opinião pública através da classificação do espectro direita e esquerda. 
Para os autores, a ideologia seria essencial na vida dos eleitores, pois dado o mundo complexo, ela seria uma ferramenta que simplificaria bastante na visão do mundo e proporcionariam aos eleitores maior facilidade de suas escolhas. Esse seria um atalho cognitivo valioso de orientação das decisões e o ajudaria a ter um framework da realidade.

Os autores acreditavam que a facilidade no uso da ideologia como um atalho cognitivo informacional serviria para o eleitor como uma forma de minimizar a complexidade das questões sociais, econômicas e políticas presentes no seu cotidiano. Assuntos esses que demandariam certo grau de sofisticação para a compreensão.

Segundo Campbell et al. (1960), a estrutura ideológica de esquerda e direita poderia ser um discernimento valioso na política, uma vez que os cidadãos seriam desinteressados e veriam a política com muita complexidade pela quantidade de assuntos em jogo. Assim, a função da ideologia seria estruturar as preferências. Ela carregaria consigo diversas estruturas de crenças em “pacotes" prontos, facilitando a análise em um ambiente que requeria alto nível de abstração devido aos determinados assuntos (CAMPBELL et. al, 1960).

Ademais, a teoria clássica do modelo espacial unidimensional de Downs (1957) argumentava que as políticas estariam dispostas ao longo do espectro ideológico de "esquerda e direita" e que os indivíduos e partidos políticos encontrariam seus pontos ideais de modo a maximizarem seu bem-estar e preferências.

Autores como Stokes (1960) e Davis et al. (1970) apontaram que a análise unidimensional downsiana falharia ao colocar o indivíduo apenas em uma única dimensão econômica, pois a realidade seria multidimensional compostas por vários aspectos diferentes e não apenas devido a questões econômicas, o indivíduo se depararia com problemas de ação coletiva que lhe acarretariam dificuldade para escolher um posicionamento ideológico.

Apesar disso, Conserve (1964) já discutia que a ideologia não era uma simples balizadora e facilitadora de como os indivíduos formariam suas opiniões acerca dos assuntos. Segundo o autor, os eleitores não usam a ideologia para se posicionarem sobre as questões, e quando se utilizam, não haveria uma conexão clara para eles sobre o que seria de esquerda e direita. Em outro momento, Converse e Pierce (1986) apontam para a falta de conhecimento do eleitor em entender o espectro esquerda e direita e que isso seria consequência do conceito variar ao longo do tempo, entre países e grupos. Portanto, podendo causar certo grau de incerteza e confusão no momento de elencar suas preferências e estruturar seu voto. Ao analisar o contexto bipartidário dos Estados Unidos, os autores concluem seus experimentos mostrando que o uso do espectro ideológico como um atalho cognitivo para as escolhas políticas é bastante baixo. 
Nesse sentido, Zechmeister (2015), ao abordar a formação de opinião pública através da ideologia, concluiu que na América Latina os eleitores não teriam identificação ideológica, pontuando baixa conexão do sentido de "esquerda e direita". Ainda segundo a autora, a ideologia para os eleitores não seria uma causa para a escolha do seu voto e, por sua vez, as possíveis variáveis determinantes poderiam ser polarização, fragmentação, competição pragmática e o clientelismo. Para Dalton (2016), muitos cidadãos estariam centrando suas escolhas levando mais em consideração grupos específicos, especialmente, o partidarismo.

No caso brasileiro, o primeiro estudo a se comprometer a mensurar o efeito da ideologia no voto foi de Singer (1999), que, ao analisar as eleições presidenciais de 1989 e 1994, apontou o efeito da ideologia (esquerda-direita) central para os eleitores na escolha do voto. O eleitor brasileiro utilizaria fortemente a ideologia para escolher seu candidato de acordo com seus interesses pessoais.

Ao adicionar a sofisticação política dos eleitores no modelo, Carreirão (2002) encontra um resultado distinto. Segundo esse autor, os eleitores formariam seu voto levando em consideração apenas o desempenho do governo e avaliação pessoal dos candidatos. Já Carreirão (2007), encontra uma correlação fraca entre identificação partidária e voto para as eleições de 2006, como também uma redução da variável ideologia como preditora do voto. Havendo, portanto, apenas uma correlação moderada nos estratos sociais de nível superior.

Por sua vez, Izumi (2019), utilizando dados do Estudo Eleitoral Brasileiro, corrobora o modelo da teoria espacial do voto, em que o eleitor tenderia a votar no candidato que está mais próximo do seu ponto de vista ideológico. Para Izumi (2019), o modelo espacial do voto parece prever razoavelmente o comportamento do eleitor para todos os níveis de sofisticação política. Sobretudo, na parcela maior dos eleitores poucos sofisticados, que tenderiam a escolher $\mathrm{o}$ candidato mais próximo a sua preferência ideológica ${ }^{4}$.

Em suma, como foi exposto, não há consenso na literatura sobre o efeito da ideologia na política e existe uma lacuna teórica sobre sua associação com percepção macroeconômica na felicidade. Para tanto, busco responder a seguinte questão: qual é o efeito da percepção macroeconômica de inflação e desemprego sobre o nível de felicidade?

\section{METODOLOGIA}

\footnotetext{
${ }^{4}$ Para maior aprofundamento desse debate ver: Holzhacker e Balbachevsky (2007), Nicolau (2014) e Amaral e Ribeiro (2015).
} 
Esta seção apresenta as técnicas, dados e variáveis de interesse a serem analisadas neste estudo multivariado. Espera-se contribuir com o princípio da replicabilidade científica para que outros pesquisadores possam chegar aos resultados esperados (KING, 2015). Os dados da amostra ( $\mathrm{n}=5.563$ ) são do Latin American Public Opinion Project (LAPOP) e correspondem ao período de 2010 a 2016 ${ }^{5}$. O intervalo dos dados de painel acontece em $2 \mathrm{em} 2$ anos a diferentes indivíduos ${ }^{6}$. As variáveis foram extraídas de perguntas fechadas do survey, no qual o eleitor, espontaneamente, assinalava as categorias que melhor expressassem sua opinião.

Por sua vez, a escolha de caso do Brasil é dada por dois grandes fatores: (1) lacuna teórica: pela falta, salvo melhor juízo, de trabalhos empíricos de opinião pública que mensurem a relação entre percepção das políticas macroeconômicas, felicidade e ideologia no Brasil; (2) saliência do tema: segundo o World Happiness Report de $2019^{7}$, elaborado pela Organização das Nações Unidas $(\mathrm{ONU})$, o Brasil perdeu quatro posições do ranking, caindo da $28^{\circ}$ posição para a $32^{\circ}$, em relação a 2018. Já o Happy Planet Index - 2016 ${ }^{8}$, construído pelo New Economics Foundation (NEF), aponta o Brasil na $23^{\circ}$ colocação com bem-estar moderado, em comparação com outros países. Portanto, esses fatores são importantes para o estudo de caso dada a saliência e importância do tema em todo o mundo e na literatura.

Como variável dependente, este trabalho utiliza a medida de "satisfação com a vida" como uma proxy da variável "nível de felicidade". Segundo Frey e Stutzer (2001), a felicidade dos indivíduos pode ser capturada quando são feitas perguntas de como eles estão satisfeitos com sua vida. Essa é uma estratégia considerada como mensuração da qualidade geral da vida e bem-estar?. Os autores colocam que uma única pergunta (direta) ou várias perguntas sobre auto relatos globais podem obter indicações da avaliação dos indivíduos sobre sua satisfação ou felicidade.

Ainda segundo os autores, "measures of subjective well-being can thus serve as proxies for "utility". Individuals' happiness or life satisfaction can be captured in large surveys."(FREY E STUTZER, 2001, 5). Portanto, para mensurar essa variável no questionário, os indivíduos responderam a seguinte questão:

\footnotetext{
5 Devido à disponibilidade das perguntas feitas sobre a variável dependente (nível de satisfação) e as variáveis econômicas (sobre inflação e desemprego)

${ }^{6}$ Os dados e perguntas do LAPOP para o Brasil utilizados neste trabalho são correspondentes aos anos de 2010, 2012, 2014 e 2016. Para acesso ao questionário e aos bancos de dados ver: < http://datasets.americasbarometer.org/database/index.php?freeUser=true $>$. Acessado em 09 de agosto de 2019.

${ }^{7}$ Para maiores informações acessar: https://s3.amazonaws.com/happiness-report/2019/WHR19.pdf. Acessado em 5 de março de 2020.

8 Para acessar a relação completa com todos os países presente do Happy Planet Index acessar: http://happyplanetindex.org/countries. Acessado em 5 de março de 2020.

${ }^{9}$ Para mais informações sobre esse debate ver Pavot e Diener (1993) e Inglehart et al. (2000).
} 
"Para começar, em geral, até que ponto o(a) sr./sra. está satisfeito(a) com sua vida? $O(A)$ sr./sra. diria que está... Leia as Alternativas]?”

Nessa pergunta, existem quatro tipos de categorias: “(1) Muito satisfeito”; “(2) Pouco Satisfeito"; “(3) Pouco insatisfeito" e "(4) Muito insatisfeito". Os casos "Não sabem” e "Não Respondeu" foram excluídos da análise. Essa variável foi transformada em dummy, em que "Muitos satisfeito" e "Pouco satisfeito" foram categorizados como "1 - Sim", e "Pouco insatisfeito" e "Muito satisfeito" como "0 - Não".

Em relação à posição ideológica, ao serem apresentados um cartão, os indivíduos eram estimulados responderem a seguinte questão:

"Agora, para mudar de assunto... Nesse cartão há uma escala, de 1 a 10, na qual o número 1 significa "esquerda" e o 10 significa "direita". Hoje em dia, quando se conversa de tendências politicas, fala-se de pessoas que simpatizam mais com a esquerda e de pessoas que simpatizam mais com a direita. De acordo com o sentido politico que os termos "esquerda" e "direita" têm para o(a) sr./ sra, onde o(a) sr./ sra. se situa nesta escala?"

Os eleitores entrevistados classificaram suas respostas nesse espectro. Seguindo a recomendação de Di Tella e MacCulloch (2005), as respostas foram classificadas do seguinte modo: os eleitores de esquerda são aqueles que sinalizaram de "1 a 4" e de direita, aqueles de "7 a 10", os demais casos foram excluídos na análise, por não serem objetos da pesquisa. A partir dessa divisão, foram construídas duas variáveis dummies, uma para indivíduos de esquerda e outra para direita.

Como variáveis independentes de percepção econômica macroeconômica de inflação e desemprego, foi feita a seguinte pergunta:

"Na sua opinião, qual é o problema mais grave que o país está enfrentando?"

Os indivíduos foram estimulados a escolher apenas uma alternativa dentre 40 categorias. Assim, classifiquei como "Inflação" os indivíduos que assinalaram as respostas "Inflação e alto preço" e "Economia, problemas com a crise". Essa junção de categoria se deu pelo baixo número de respondentes à primeira e por ambos os assuntos estarem correlacionados nas respostas. Essa estratégia pode ser adotada dada a incipiência dos respondentes em distinguir ambos os assuntos (DI TELLA, MAC CULLOCH E OSWALD, 1997). Assim, foi criada uma variável dummy de 
"Inflação", em que "1 - Sim", quando a inflação é o maior problema e "0 - Não", quando não. Por sua vez, a categoria "Desemprego" também foi categorizada em uma variável dummy, em que "1 Sim", quando esse assunto é o maior problema, e " 0 - Não". As demais categorias foram excluídas na análise.

Seguindo as recomendações da literatura em inserir variáveis com características microeconômicas, foram utilizadas variáveis de controle que dizem respeito à percepção do eleitor sobre a economia no âmbito geral e individual. Para mensurar essas dimensões, foi utilizada a seguinte pergunta:

"Agora, falando da economia...Como o(a) sr./sra. avalia a situação econômica do país?"

As categorias para essa pergunta eram "Melhor", "Igual” e "Pior". Para análise, "Não sabe" ou "Não Responderam" foram excluídas. Assim, foi criada uma variável qualitativa contendo 3 categorias, sendo "1 - Melhor", "2 - Igual” e "3 - Pior".

O mesmo processo ocorreu para quando perguntado sobre situação econômica individual:

" $O(A)$ sr./sra. acha que sua atual situação econômica está melhor, igual ou pior que a de bá doze meses?"

As respostas para essa pergunta eram "Melhor", "Igual" e "Pior". Analiticamente, "Não sabe" ou "Não Responderam" foram excluídos da análise. Assim, foi criada outra variável qualitativa contendo 3 categorias: "1 - Melhor", "2 - Igual” e "3 - Pior".

Por fim, para mensurar o efeito do gênero, foi utilizada a variável "homem" (assumindo valor de "0" - Não e "1" Sim). Nessa pergunta, o próprio aplicador preenchia a resposta. Ademais, os dados omitidos das variáveis do banco de dados final foram excluídos.

Pela variável dependente ser binária aconselha-se o uso da Regressão Logística (HAIR et al., 2009). Por sua vez, como a hipótese do trabalho é interativa, de que o efeito da percepção da inflação e do desemprego sobre o nível felicidade depende da ideologia política no Brasil, Brambor et al. (2006) recomendam que nesse caso sejam inclusos todos os termos interativos e suas especificações no modelo. Assim, serão elaborados 5 modelos Logit para cada cenário de interação.

Os dois primeiros modelos estimarão o efeito dos indivíduos da ideologia de "esquerda" sobre a felicidade (sem e com interação). O modelo 1 testará apenas a percepção do desemprego para este grupo na VD, controlada pelas demais variáveis. Tendo o seguinte modelo: 


$$
\begin{gathered}
\operatorname{Pr}\left(\text { Felicidade }=1 \mid x_{i}\right)=p\left(\beta 0+\beta_{1} \text { Esq }+\beta_{2} \text { Inf }+\beta_{3} \text { Des }+\beta_{4} H+\beta_{5} E G \_ \text {igual }+\beta_{6} \text { EG_pior }+\right. \\
\left.\beta_{7} \text { EP_igual }+\beta_{8} \text { EP_pior }+\epsilon\right)
\end{gathered}
$$

Onde, a variável dependente " $\operatorname{Pr}($ Felicidade $=1)$ " é função do eleitor ser de Esquerda $\left(\boldsymbol{\beta}_{1}\right.$ Esq), acreditar que a inflação é o maior problema ( $\left.\boldsymbol{\beta}_{2} \mathbf{I n f}\right)$, acreditar que o desemprego é o maior problema $\left(\boldsymbol{\beta}_{3}\right.$ Des$)$, controlado por ser do sexo masculino $\left(\boldsymbol{\beta}_{4} \mathbf{H}\right)$, ter percepção que a economia do país está na mesma ( $\boldsymbol{\beta}_{5} \mathbf{E} \mathbf{G} \_$igual) ou pior $\left(\boldsymbol{\beta}_{6} \mathbf{E} \mathbf{G} \_\right.$pior) e que a sua economia pessoal está na mesma $\left(\boldsymbol{\beta}_{7} \mathbf{E P} \_\right.$igual $)$ou pior $\left(\boldsymbol{\beta}_{8} \mathbf{E P} \_\right.$pior $)$. $a$ corresponde ao intercepto e $\in$ ao erro do modelo de regressão. Espera-se que a percepção de desemprego tenha um efeito negativo no nível de felicidade.

O modelo 2 será adicionado à interação entre ser de esquerda e acreditar que o desemprego é o maior problema ( $\beta_{2}$ Esq*Des), mantendo as demais variáveis. Espera-se desse modelo que a variável de interação tenha um impacto negativo no nível de felicidade. Assim, temos o modelo 2:

$$
\begin{aligned}
\operatorname{Pr}\left(\text { Felicidade }=1 \mid x_{i}\right)= & p\left(\beta 0+\beta_{1} \text { Esq }+\beta_{2} \text { Esq } * \text { Des }+\beta_{3} \text { Inf }+\beta_{4} \text { Des }+\beta_{5} H+\beta_{6} \text { EG_igual }+\right. \\
& \left.\beta_{7} \text { EG_pior }+\beta_{8} E P \_ \text {igual }+\beta_{9} \text { EP_pior }+\epsilon\right)
\end{aligned}
$$

Os dois seguintes modelos, testarão a percepção sobre inflação para o grupo de indivíduos mais à direita nos níveis de felicidade (sem e com interação). O modelo 3, procura apenas estimar o efeito da percepção de inflação sobre a VD, controlada pelas demais VC.

$$
\begin{gathered}
\operatorname{Pr}\left(\text { Felicidade }=1 \mid x_{i}\right)=p\left(\beta 0+\beta_{1} \text { Dir }+\beta_{2} \text { Inf }+\beta_{3} \text { Des }+\beta_{4} H+\beta_{5} \text { EG_igual }+\beta_{6} \text { EG_pior }+\right. \\
\left.\beta_{7} \text { EP_igual }+\beta_{8} \text { EP_pior }+\epsilon\right)
\end{gathered}
$$

A variável dependente " $\operatorname{Pr}\left(\right.$ Felicidade =1)" é função do eleitor ser de Direita ( $\boldsymbol{\beta}_{1}$ Dir), acreditar que a inflação é o maior problema ( $\left.\boldsymbol{\beta}_{2} \mathbf{I n f}\right)$, acreditar que o desemprego é o maior problema $\left(\beta_{3}\right.$ Des), controlado por ser do sexo masculino $\left(\beta_{4} \mathbf{H}\right)$, ter percepção que a economia do país está na mesma ( $\boldsymbol{\beta}_{5} \mathbf{E}$ G_igual) e pior $\left(\boldsymbol{\beta}_{6} \mathbf{E} \mathbf{G} \_\right.$pior) e que a sua economia pessoal está na mesma $\left(\boldsymbol{\beta}_{7} \mathbf{E P} \_\right.$igual) ou pior $\left(\boldsymbol{\beta}_{8} \mathbf{E P} \_\right.$pior). a corresponde ao intercepto e $\in$ ao erro do modelo de regressão. Espera-se que a percepção de inflação reduza o nível de felicidade dos eleitores.

O modelo 4 adicionará à interação entre ser de direita e acreditar que a inflação é o grande problema $\left(\boldsymbol{\beta}_{2}\right.$ Dir*Inf). Espera-se que essa variável reduza a chance no nível de felicidade dos indivíduos desses eleitores. Temos, portanto, a seguinte equação: 


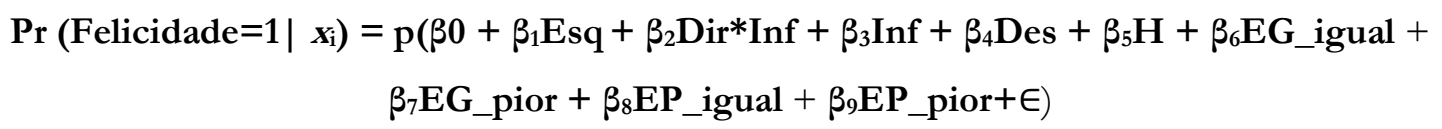

Já no modelo 5, serão adicionadas todas as variáveis independentes (sem e com interação), assim como, suas respectivas variáveis de controle. Todos os modelos buscarão testar os possíveis cenários de como as variáveis independentes impactam nos níveis de felicidade dos eleitores brasileiros.

Os testes de robustez e capacidade preditiva das Regressões Logísticas seguiram as recomendações de Garrett (2008) e Ferreira et al. (2015) sobre a performance de um modelo logit $t^{10}$. Por fim, a análise dos dados foi por meio do software estatístico R Studio (versão 3.3), e visando a replicabilidade, tanto o banco de dados quanto os scripts produzidos, estão adicionados em repositório público no GitHub ${ }^{11}$.

\section{RESULTADOS ${ }^{12}$}

Testando a hipótese de pesquisa de que o efeito da percepção e inflação e desemprego no nível de felicidade depende da ideologia política no Brasil, foram estimados 5 modelos logit para cada ideologia, com e sem interação, e um modelo contendo todas as variáveis. A tabela 1 sumariza os principais achados dos modelos interativos da Regressão Logística.

Tabela 1 - Modelos de Regressão Logística ${ }^{13}$

\begin{tabular}{|c|c|c|c|}
\hline \multirow{2}{*}{ Variáveis } & Esquerda & Direita & $\begin{array}{l}\text { Todos } \\
\text { (5) }\end{array}$ \\
\hline & $\begin{array}{c}\text { Com } \\
\text { Interação } \\
\text { (2) }\end{array}$ & $\begin{array}{c}\text { Com } \\
\text { Interação } \\
\text { (4) }\end{array}$ & \\
\hline Constante & $\begin{array}{c}2,593 * * * \\
(0,127)\end{array}$ & $\begin{array}{c}2,609 * * * \\
(0,130)\end{array}$ & $\begin{array}{c}2,686 * * * \\
(0,141)\end{array}$ \\
\hline Esquerda & $\begin{array}{l}-0,090 \\
(0,104)\end{array}$ & & $\begin{array}{l}-0,164 \\
(0,119)\end{array}$ \\
\hline
\end{tabular}

\footnotetext{
${ }^{10}$ Serão feitos os Teste de Concordância, Sensitividade, Especificidade, Erro de Classificação Incorreta e de Receiver Operating Characteristics Curve (ou Curva ROC) para verificar as seguranças de predição dos modelos.

${ }^{11}$ Para acessar o banco de dados e scripts na análise de dados acessar: < https://github.com/andersonheri/AndersonHenrique-ad-ufpe-2019 > . Acessado em 06 de novembro de 2019.

${ }^{12} \mathrm{~A}$ análise descritiva encontra-se no final do artigo.

${ }^{13}$ Os testes de robustez dos modelos logit sem interação, assim como, os modelos de Regressão Logística sem as interações encontram-se no Apêndice.
} 
HENRIQUE, A. Percepsão macroeconômica, felicidade e ideologia: um estudo de caso do brasil (2010-2016). DOI: 10.5380/recp.v\%ovi\%i.70099

\begin{tabular}{|c|c|c|c|}
\hline Direita & & $\begin{array}{l}-0,098 \\
(0,099)\end{array}$ & $\begin{array}{l}-0,185 \\
(0,114)\end{array}$ \\
\hline Perc. de Inflação & $\begin{array}{l}-0,003 \\
(0,167)\end{array}$ & $\begin{array}{l}-0,106 \\
(0,196)\end{array}$ & $\begin{array}{l}-0,098 \\
(0,197)\end{array}$ \\
\hline Perc. de Desemprego & $\begin{array}{c}-0,542 * * * \\
(0,146)\end{array}$ & $\begin{array}{c}-0,546 * * * \\
(0,117)\end{array}$ & $\begin{array}{c}-0,535^{* * *} \\
(0,146)\end{array}$ \\
\hline Homem & $\begin{array}{c}0,197 * * \\
(0,090)\end{array}$ & $\begin{array}{c}0,198^{* *} \\
(0,090)\end{array}$ & $\begin{array}{c}0,202^{* *} \\
(0,090)\end{array}$ \\
\hline Ec. Geral - Igual & $\begin{array}{c}0,057 \\
(0,131)\end{array}$ & $\begin{array}{c}0,051 \\
(0,131)\end{array}$ & $\begin{array}{l}0,051 \\
(0,131)\end{array}$ \\
\hline Ec. Geral - Pior & $\begin{array}{c}-0,373 * * * \\
(0,129)\end{array}$ & $\begin{array}{c}-0,378^{* * *} \\
(0,129)\end{array}$ & $\begin{array}{c}-0,371 * * * \\
(0,129)\end{array}$ \\
\hline Ec. Individual - Igual & $\begin{array}{c}0,043 \\
(0,123)\end{array}$ & $\begin{array}{c}0,048 \\
(0,123)\end{array}$ & $\begin{array}{c}0,052 \\
(0,123)\end{array}$ \\
\hline Ec. Individual - Pior & $\begin{array}{c}-0,904 * * * \\
(0,123)\end{array}$ & $\begin{array}{c}-0,915^{* * *} \\
(0,123)\end{array}$ & $\begin{array}{c}-0,914 * * * \\
(0,124)\end{array}$ \\
\hline $\begin{array}{c}\text { Esquerda*Desempre } \\
\text { go }\end{array}$ & $\begin{array}{l}-0,011 \\
(0,239)\end{array}$ & & $\begin{array}{l}-0,029 \\
(0,240)\end{array}$ \\
\hline Direita*Inflação & & $\begin{array}{c}0,331 \\
(0,355)\end{array}$ & $\begin{array}{c}0,331 \\
(0,355)\end{array}$ \\
\hline Observações & 5,563 & 5,563 & 5,563 \\
\hline Log-Likelihood & $-1.779,165$ & $-1.778,908$ & $-1.777,716$ \\
\hline AIC & $3.578,330$ & $3.577,817$ & $3.579,432$ \\
\hline
\end{tabular}

Como podemos observar, o modelo 2 de interação com o grupo da Esquerda apresenta o Log-Likelihood de -1.779,165 e AIC de 3.578,33. Estimando a razão de chance, transformando os odd ratios em probabilidade ${ }^{14}$ e controlando pelas demais variáveis, temos que a variável do termo interativo entre ser de esquerda e ter uma percepção de inflação como principal problema não apresenta significância estatística. Ou seja, essa não afeta o nível de felicidade dos indivíduos.

Quando temos um modelo de Regressão Logística, Brambor et al. (2006) indicam também calcular o efeito marginal e erro padrão dos temos interativos, para $Z=0$ (variável sem interação) e $Z=1$ (variável com interação), além da análise gráfica. A tabela 2 abaixo sumariza essa informação.

Tabela 2 - Efeito Marginal e Erro Padrão do Termo Interativo no Modelo 2

\begin{tabular}{c|c|c}
\hline \hline Termo de Interação & $\mathbf{Z}=\mathbf{0}$ (sem o termo) & $\mathbf{Z = 1}$ (com o termo) \\
\hline Efeito Marginal de X & $\begin{array}{c}\text { Desemprego } \\
(-0,056) * * *\end{array}$ & $\begin{array}{c}\text { Desemprego*Esquerda } \\
(-0,0009)\end{array}$ \\
\hline Erro Padrão & 0,009 & 0,020 \\
\hline \hline
\end{tabular}

${ }^{14} \mathrm{~A}$ fórmula para transformar os odd ratios em razão de chance é: $\mathrm{p}=\frac{\operatorname{Exp}(\beta)}{\operatorname{Exp}(\beta)+1}$ 
Fonte: Elaboração do autor (2019).

Analisando o efeito marginal do termo interativo, temos que a variável independente "Desemprego", quando não interagida do modelo, tem Efeito Marginal de -0,056 estatisticamente significativo ( $\mathrm{p}$-valor<0,05) e Erro Padrão de 0,009. Por outro lado, quando observamos o termo interativo do modelo, o Efeito Marginal desaparece.

O modelo 4 com o grupo de Direita tem Log-Likelihood de -1.778,908 e AIC de 3.577,817. Estimando as chances do nível de felicidade, a variável de interação entre ser de direita e abordar que a inflação como o maior problema do país, controlando pelas demais VC, não apresenta significância estatística. Analisando o efeito marginal do termo interativo presente no modelo 4. Temos a seguinte configuração presente na tabela 3:

Tabela 3 - Efeito Marginal e Erro Padrão do Termo Interativo no Modelo 4

\begin{tabular}{c|c|c}
\hline \hline Termo de Interação & $\mathbf{Z}=\mathbf{0}$ (sem o termo) & $\mathbf{Z}=\mathbf{1}$ (com o termo) \\
\hline Efeito Marginal de X & $\begin{array}{c}\text { Inflação } \\
(-0,009)\end{array}$ & $\begin{array}{c}\text { Inflação*Direita } \\
(0.024)\end{array}$ \\
\hline Erro Padrão & 0,009 & 0,020 \\
\hline \hline & $\begin{array}{l}\text { Nota: }{ }^{*} \mathrm{p}<0.1 ; * * \mathrm{p}<0.05 ; * * * \mathrm{p}<0.01 \\
\text { Fonte: Elaboração do autor }(2019) .\end{array}$
\end{tabular}

Diferentemente do modelo 2, a variável com e sem interação não apresentam efeitos marginais estatisticamente significativos sobre a variável dependente. Como o foco principal é avaliar o comportamento das variáveis com interação para os dos modelos, ambos não produzem efeito algum sobre o nível de felicidade ${ }^{15}$.

Por fim, o modelo 5 com todas variáveis, assim como os outros anteriores, apenas a percepção do desemprego (reduz 35\%), sexo masculino (aumenta 16\%) percepção com a economia geral do país (reduz 27\%) e percepção de sua economia (reduz 47\%) impactaram a variável dependente (modelo com um Log-Likelihood de -1.777,716 e AIC de 3.579,432).

Assim, com base nos modelos, podemos concluir que a percepção dos indivíduos de inflação e o desemprego, quando levada em consideração a ideologia política, não afetar seus níveis de felicidade no Brasil. Entretanto, os resultados reforçam as conclusões da literatura estrangeira sobre a insuficiência da ideologia como um importante atalho cognitivo.

\section{CONSIDERAÇÕES FINAIS}

${ }^{15}$ Os gráficos do efeito marginal das interações encontram-se no Apêndice. 
Como pode ser visto, a felicidade é um assunto que está ganhando destaque nos estudos da Ciência Política e da Economia. Os trabalhos na área buscam estimar modelos para prever como as políticas econômicas dos governos no poder podem impactar no bem-estar dos eleitores e no estímulo em votar.

O conhecimento clássico de opinião pública acreditava que a ideologia seria essencial na vida dos eleitores, pois, dado a complexidade do mundo, ela seria uma ferramenta que simplificaria bastante visão do mundo e facilidade de escolha (CONVERSE, 1964). Zechmeister (2015) e Dalton (2016) encontraram que, dada a quantidade e complexidade dos assuntos, os eleitores buscariam outras formas de tomar suas decisões, como o partidarismo, influência familiar e determinados grupos sociais para ajudar em suas escolhas políticas. Em outros contextos, como a América Latina, essa conexão seria inexistente (ZECHMEISTER, 2015).

Por sua vez, inicialmente no Brasil, acreditava-se que a ideologia seria um ponto central na escolha do voto (SINGE, 1999), sendo rebatido por Carreirão (2002; 2007), que encontrou uma relação fraca entre estrutura ideológica e escolha do voto nas eleições presidenciais. É apenas com Izumi (2019), ao adicionar novamente a importância da sofisticação política, que a ideologia parece corroborar com a tese do modelo espacial do voto em que o eleitor brasileiro votaria em candidatos mais próximos as suas preferências.

O principal achado deste trabalho, ao estimar os modelos de Regressão Logística, é de que a percepção de problemas macroeconômicos, levando em consideração a ideologia dos indivíduos, não impacta na felicidade subjetiva dos eleitores no Brasil. Portanto, corroborando com os trabalhos anteriores que apontam a ineficiência da variável ideológica como um possível atalho cognitivo de escolhas políticas e comportamentos.

Podem existir outros fatores importantes relevantes que estejam interferindo nesse processo. Por exemplo, destaco a crescente polarização política, alta fragmentação partidária, competição pragmática entre os partidos, que dificulta a escolha do candidato, e o advento das práticas clientelistas, que facilita as trocas a nível individual, muitas vezes desconsiderando o plano macro.

Este trabalho apresenta limitações, que não comprometem os resultados apresentados. Por se tratar de dados de percepção, pode haver problema dos eleitores em distinguir certos tipos de assuntos, sobretudo, macroeconômico que exige maior sofisticação cognitiva.

\section{REFERÊNCIAS}


ALESINA, A. (1987). Macroeconomic policy in a two-party system as a repeated game. The quarterly journal of economics, vol. 102, $\mathrm{n}^{\circ}$ 3, p. 651-678.

AMARAL, O., RIBEIRO, P. (2015). Por que Dilma de novo? Uma análise exploratória do Estudo Eleitoral Brasileiro de 2014. Revista de Sociologia e Politica, vol. 23, nº 56, p. 107-123.

BRAMBOR, T., CLARK, W. R., GOLDER, M. (2006). Understanding interaction models: Improving empirical analyses. Political analysis, vol. 14, n 1, p. 63-82.

CAMPBELl, A., CONVERSE, P. E., MILlER, W. E., STOKES, D. E. (1960). The American Voter. New York: John Wiley.

CARREIRÃO, Y. (2002). A decisão do voto nas eleições presidenciais brasileiras. Rio de Janeiro: Editora FGV.

CARREIRÃO, Y. (2007). Identificação ideológica, partidos e voto na eleição presidencial de 2006. Opinião Pública, vol. 13, n 2, p. 307-339.

CAVALCANTI, T. V. de V., GUIMARÃES, J. F., NOGUEIRA, J. R. (2009). Is Brazil the Land of Happiness? Comparative Study Using a Sample with Economics Students from UFPE and Purdue. Brazilian Review of Econometrics, vol. 29, n 1, p. 17-35.

CHAPPELL, H., HAVRILESKY, T., MCGREGOR, R. (1993). Partisan Monetary Policies: Presidential Influence through the Power of Appointment, Quarterly Journal of Economics, vol. 108, $\mathrm{n}^{\circ} 1$, p. $185-218$.

CONVERSE, P. E. (1964). The Nature of Belief Systems in Mass Publics. In David E. Apter, ed., Ideology and Discontent, 206-261. Glencoe, IL: Free Press.

CONVERSE, P. E., PIERCE, R. (1986). Political Representation in France. Cambridge, MA: Belknap.

CORBI, R. B., MENEZES-FILHO, N. A. (2006). Os Determinantes Empíricos da Felicidade no Brasil. Revista de Economia Política, vol. 26, n 4, p. 518-536.

DALTON, R. (2016). Party Identification and Its Implications. Oxford Research Encyclopedia of Politics.

DI TELLA, R., MACCUllOCH, R., OSWALD, A. (2001). Preferences over Inflation and Unemployment: Evidence from Surveys of Happiness, American Economic Review, vol. 91, n 1, p. 335-341.

DI TELLA, R., MACCULLOCH, R., OSWALD, A. (2003). The Macroeconomics of Happiness. The Review of Economics and Statistics, vol. 85, $\mathrm{n}^{\circ}$. 4, p. 809-827.

DI TELLA, R., MACCULLOCH, R. (2005). Partisan social happiness. The Review of Economic Studies, vol. 72, n 2, p. 367-393.

FREY, B. S., STUTZER, A. (2002). What can economists learn from happiness research? Journal of Economic literature, vol. 40, $\mathrm{n}^{\circ}$ 2, p. 402-435. 
FREY, B. S., SCHNEIDER, F. (1978a). An empirical study of politico-economic interaction in the United States. The Review of Economics and Statistics, p. 174-183.

FREY, B. S., SCHNEIDER, F. (1978b). A politico-economic model of the United Kingdom. The Economic Journal, vol. 88, n 350, p. 243-253.

GARRET, P. E., LASKY, F. D., MEIER, K. L. (2008). Use Protocol For Evaluation of Qualitative Teste Performance, Second Edition. EP12 -A2, vol. 28, nº 3.

GOLDEN, D., POTERBA, J. (1980). The Price of Popularity: The Political Business Cycle Reexamined, American Journal of Political Science, vol. 24, n 4, p. 694-714.

HAIR, J. F. et al. (2009). Análise multivariada de dados. Bookman Editora.

HIBBS, D. (1987). The American Political Economy: Macroeconomics and Electoral Politics (Cambridge, MA: Harvard University Press).

HOLZHACKER, D., BALBACHEVSKY, E. (2007). Classe ideologia e política: uma interpretação dos resultados das eleições de 2002 e 2006. Opinião Pública, vol. 13, nº 2, p. 283-306.

INGLEHART, R., et al. (2000). World Values Surveys and Surveys and European Values Surveys, 1981-1984, 1990-1993, and 1995-1997. ICPSR version. Ann Arbor, MI: Institute for Social Research, 1999. Ann Arbor, MI: Inter-university Consortium for Political and Social Research.

ISLAM, G., WILLS-HERRERA, E. HAMILTON, M. (2009). Objective and Subjective Indicators of Happiness in Brazil: The Mediating Role of Social Class. Journal of Social Psychology, vol. 149, $\mathrm{n}^{\circ}$ 2, p. 267-272.

IZUMI, M. Y. (2019). Ideologia, sofisticação política e voto no Brasil. Opinião Pública, vol. 25, n 1 , p. 29-62.

LUZ, T. C. B. et al. (2011). Satisfaction with the neighborhood environment and health in older elderly: cross-sectional evidence from the Bambuí cohort study of aging. Cadernos de Saúde Pública, vol. 27 , p. s390-s39.

NICOLAU, J. (2014). Determinantes do voto no primeiro turno das eleições presidenciais brasileiras de 2010: uma análise exploratória”. Opinião Pública, vol 20, nº 3, p. 311-325.

PAVOT, W., DIENER, E. (1993). The Affective and Cognitive Context of Self-Reported Measures of Subjective Well-Being. Social Indicators Research, vol. 28, n 1, p. 1-20.

PINTO, S., BENCSIK, P., CHULUUN, T., GRAHAM, C. (2019). Presidential Elections, Divided Politics, and Happiness in the U.S, Working Papers. 2019-015, Human Capital and Economic Opportunity Working Group.

RABIN, M. (1998). Psychology and Economics, Journal of Economic Literature, vol. 36, p. 11-46. 
HENRIQUE, A. Percepsão macroeconômica, felicidade e ideologia: um estudo de caso do brasil (2010-2016). DOI: 10.5380/recp.v\%ovi\%i.70099

RIBEIRO, C. A. C. (2015). Renda, Relações Sociais e Felicidade no Brasil. Dados-Revista de Ciências Sociais, vol. 58, n 1, p. 37-78.

SINGER, A. (1999). Esquerda e direita no eleitorado brasileiro: a identificação ideológica nas disputas presidenciais de 1989 e 1994. São Paulo: Edusp.

SINGH, S. P. (2014). Not all election winners are equal: Satisfaction with democracy and the nature of the vote. European Journal of Political Research, vol. 53, n 2, p. 308-327.

STOKES, D. E. (1963). Spatial Models of Party Competition. American Political Science Review, vol. $57, \mathrm{n}^{\circ} 2$, p. $368-377$.

ZECHMEISTER, E. J. (2015). Left-right identifications and the Latin American voter. The Latin American voter: Pursuing representation and accountability in challenging contexts, p. 195-225.

\section{APÊNDICE}

Gráfico 1 - Frequência de Respostas das Variáveis Sexo, Ideologia e Felicidade
Sexo
Ideologia
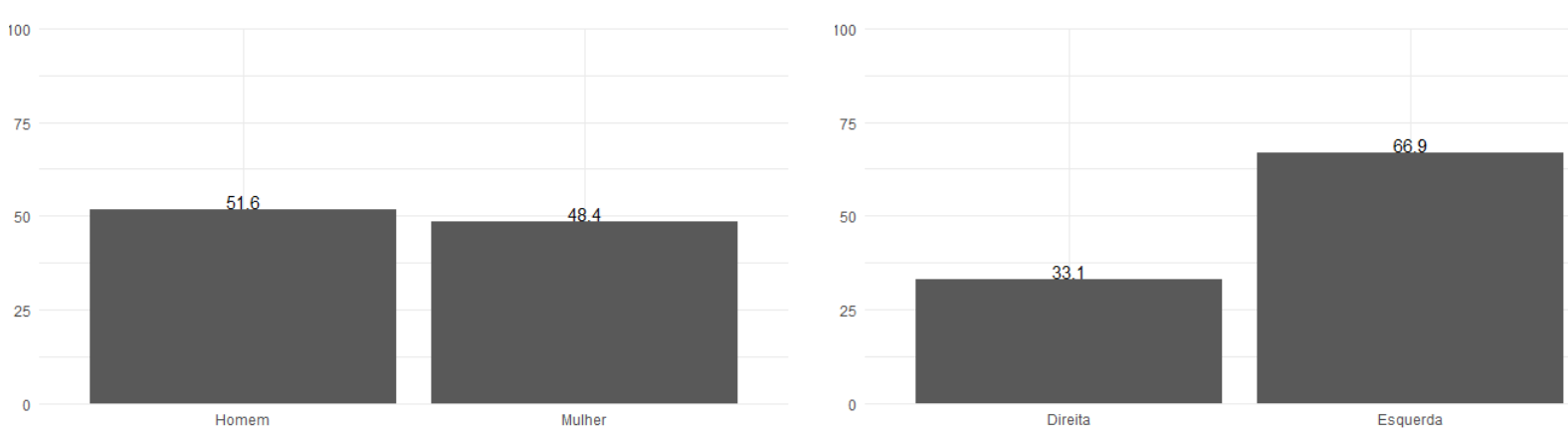

Felicidade

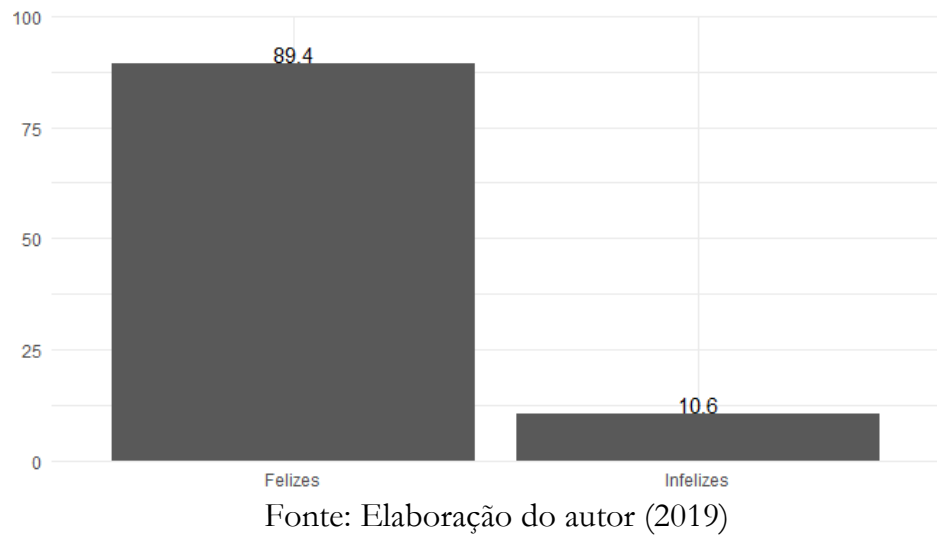

Tabela 1 - Quantidade da Percepção de Problemas Macroeconômicos de Inflação e Desemprego 
HENRIQUE, A. Percepsão macroeconômica, felicidade e ideologia: um estudo de caso do brasil (2010-2016). DOI:

\begin{tabular}{c|c|c}
\hline \hline Variável & Quantidade & Porcentagem \\
\hline Inflação & 387 & 36,57 \\
\hline Desemprego & 671 & 63,43 \\
\hline Total & 1.058 & $100 \%$ \\
\hline \hline \multicolumn{2}{r}{ Fonte: Elaboração do autor (2019). }
\end{tabular}

Tabela 2 - Modelo de Regressão Logística sem os termos interativos

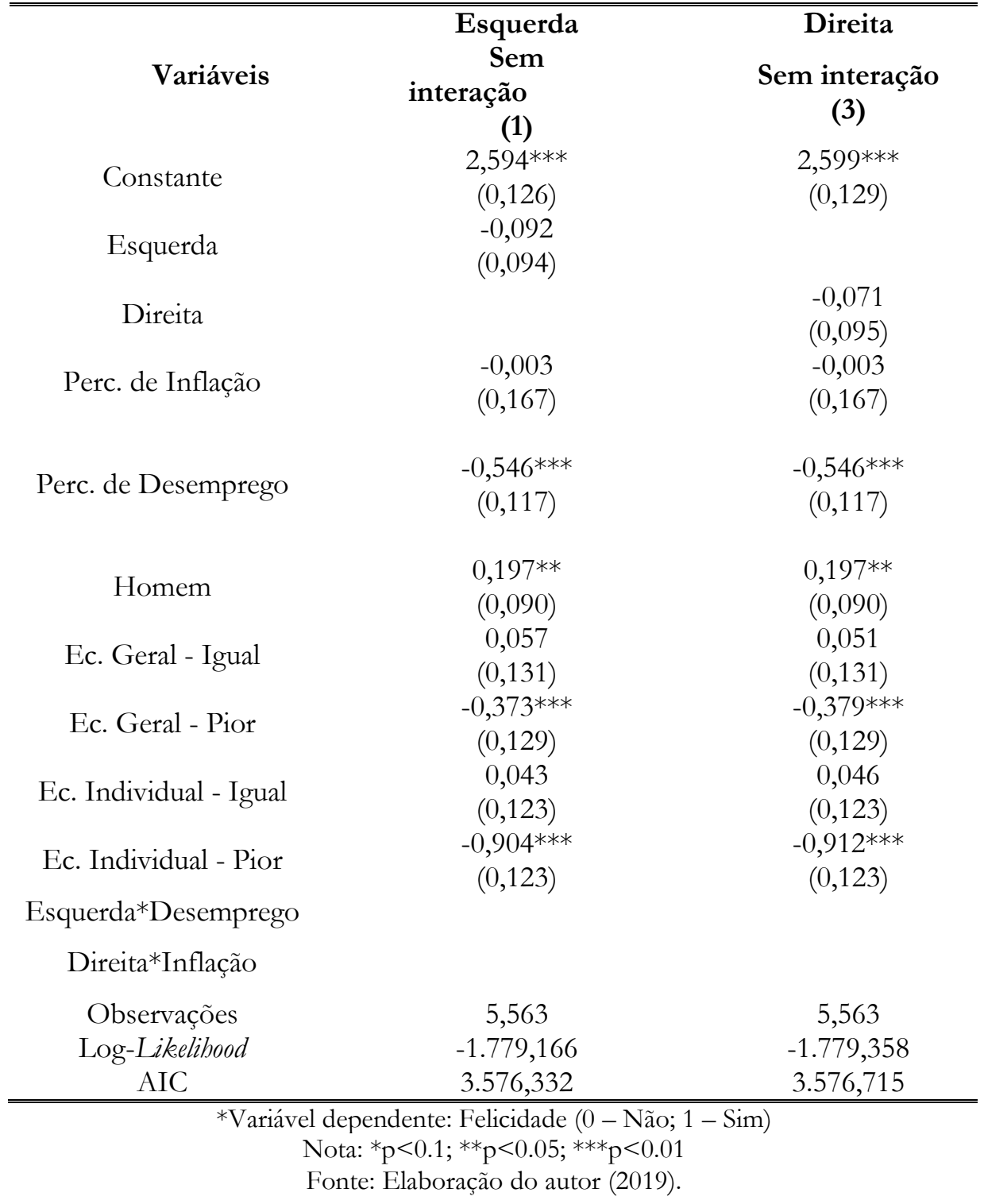

Tabela 3 - Testes de Robustez e Capacidade Preditiva dos Modelos de Regressão Logística

\begin{tabular}{l|c|c|c|c}
\hline \hline Modelos & $\begin{array}{c}\text { Testes de } \\
\text { Concordância }\end{array}$ & $\begin{array}{c}\text { Erro de } \\
\text { Classificação } \\
\text { Incorreta }\end{array}$ & Especificidade & Sensibilidade \\
\hline \hline
\end{tabular}


HENRIQUE, A. Percepção macroeconômica, felicidade e ideologia: um estudo de caso do brasil (2010-2016). DOI: 10.5380/recp.v\% \%i \%i.70099

\begin{tabular}{c|c|c|c|c}
\hline \hline $\begin{array}{c}\text { Modelo (1) - } \\
\text { Sem Interação }\end{array}$ & 0,649 & 0,108 & 0,025 & 0,993 \\
\hline $\begin{array}{c}\text { Modelo (3) - } \\
\text { Sem Interação }\end{array}$ & 0,651 & 0,106 & 0,010 & 0,997 \\
\hline $\begin{array}{c}\text { Modelo (5) - } \\
\text { Todos }\end{array}$ & 0,657 & 0,109 & 0,035 & 0,990 \\
\hline \hline
\end{tabular}

Fonte: Elaboração do autor (2019).

Gráfico 2 - Curva ROC dos Modelos de Regressão Logísticas

Modelo 1 - Esquerda (Sem interação)

ROC Curve

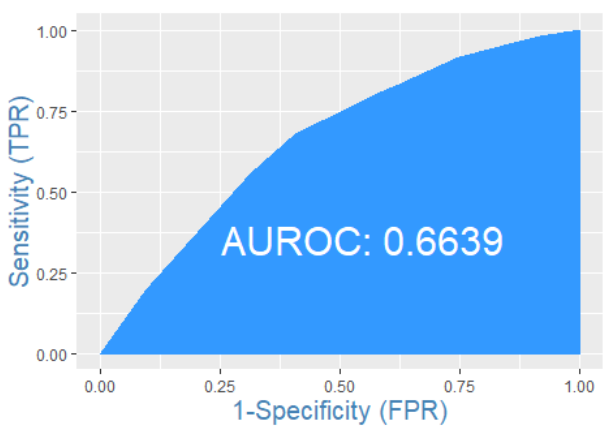

Modelo 3 - Direita (Sem interação)

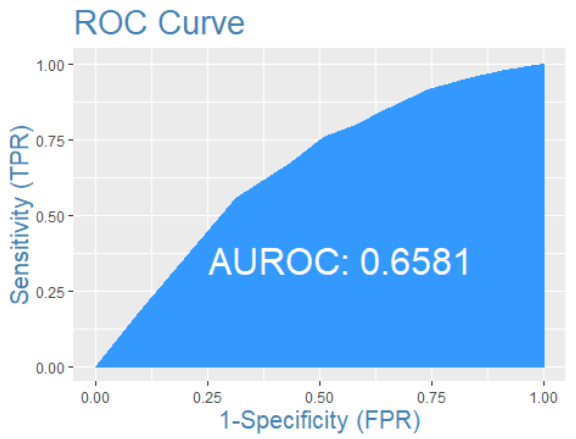

Modelo 2 - Esquerda (Com interação)

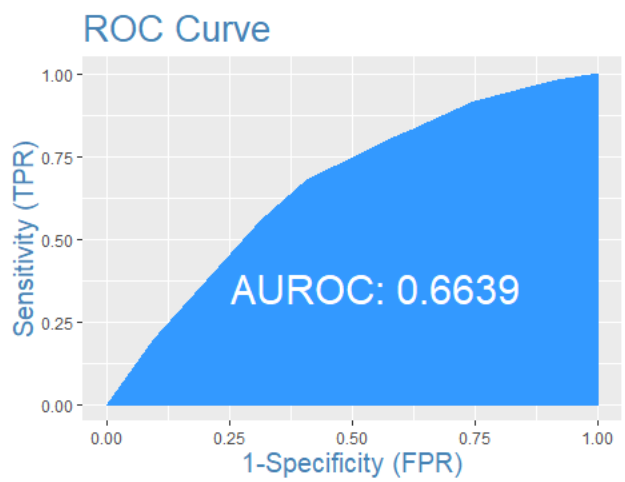

Modelo 4 - Direita (Sem interação)

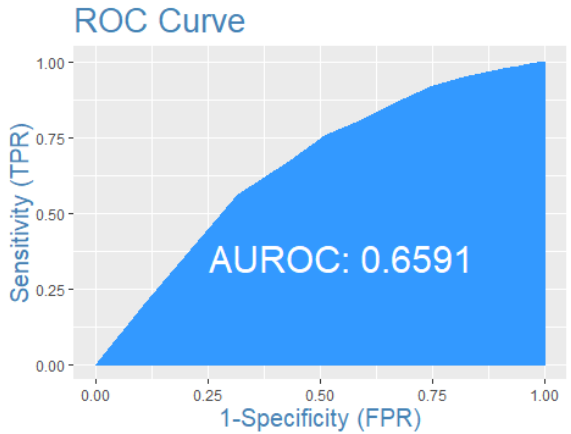

Modelo 5 - Todos

ROC Curve

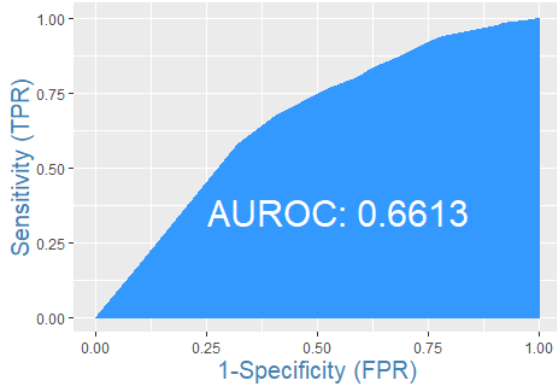

Fonte: Elaboração do autor (2019). 
HENRIQUE, A. Percepção macroeconômica, felicidade e ideologia: um estudo de caso do brasil (2010-2016). DOI: $10.5380 /$ recp.v\%vi\% \%i.70099

Gráfico 3 - Efeito Marginal dos Termos Interativos dos Modelos 2 e 5

Modelo 2 - Efeito Marginal termo interativo da Esquerda e percepção de Desemprego na Felicidade

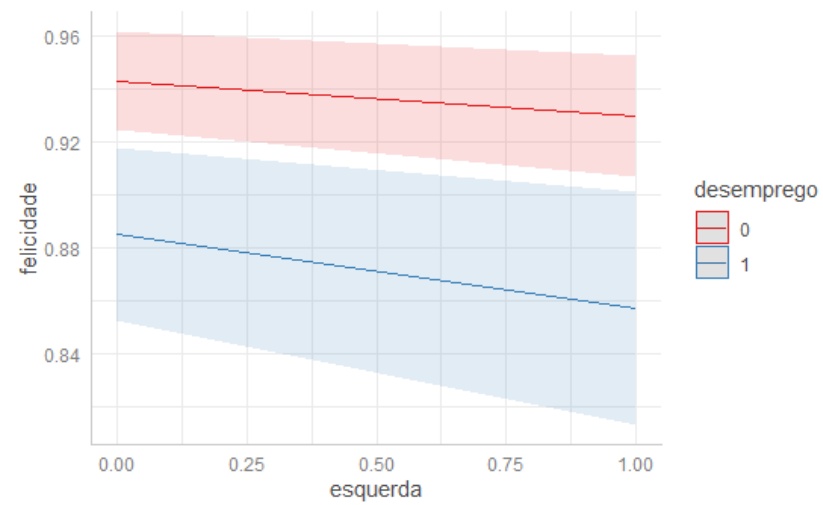

Fonte: Elaboração do autor (2019).
Modelo 4 - Efeito Marginal do termo interativo de Direita e percepção de Inflação na Felicidade

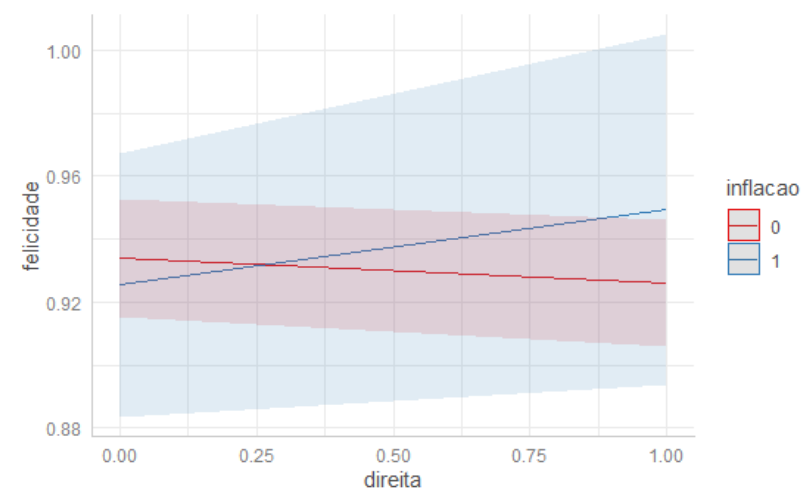

